\title{
Beware of Over-Enthusiasm for CT Scans!
}

\author{
Biplab Mishra* \\ All India Institute of Medical Sciences (AIIMS), India
}

Submission: June 23, 2016; Published: June 29, 2016

*Corresponding author: Biplab Mishra, All India Institute of Medical Sciences (AIIMS), 304, JPNATC, AIIMS, New Delhi, India, Tel: 8010135279; Email: biplabaiims@gmail.com

\begin{abstract}
The problem of over use of CT scans has become an epidemic in our present society and it is going to worsen in coming years if appropriate! steps are not taken now. This has become a complex issue with interplay of many factors like doctor factors, patient and public factors, action/ inaction of monitoring bodies and company driven. The issue simply is non-judicious use of CT scans. There is no doubt about CT scans being one of the most useful tools in diagnostic radiology. Unfortunately, doctors are now advising CT scans just at the drop of hat and history and clinical examination have taken not only a back seat but not even considered wisely in many cases. The aim of this editorial is to spread awareness among doctors about the wise use of CT scans or at least to avoid over-enthusiasm about it highlighting radiation, contrast induced nephropathy (CIN), hypersensitivity to contrast mediums and cost factor.
\end{abstract}

\section{Introduction}

One of the maladies of our present society is that we have become too 'clever' with technology but at the same time we have become 'fools' enough to get enslaved by them. Likewise this editorial is about how we think CT scan helps us and at the same time how we choose to turn a blind eye to what harm it may do! The issue simply is non-judicious use of CT scans. There is no doubt about CT scans being one of the most useful tools in diagnostic radiology. They provide a noninvasive and quick modality for doctors to assess anatomy from head to toe in different medical conditions. Therefore their use has increased dramatically over the last decade and is still increasing especially with the refinement of the technology giving more anatomical information than ever before, thus driving both doctors' and patients' demands. Unfortunately, with this has come a peculiar situation where doctors are now advising CT scans just at the drop of hat and history and clinical examination have taken not only a back seat but not even considered wisely in many cases.

Recently, I encountered a case where a patient with a diagnosis of cholecysto-colic fistula developed a post operative wound discharge following right subcostal laparotomy (for cholecystectomy + resection of colonic segment with end to end anastomosis) with no clinical or laboratory findings suggestive of colonic leak. Still a professor went on to carry out a CT scan on this patient solely based on fecal like material discharging from the wound overlooking the clinical (history and examination) and laboratory findings, where not a single finding was pointing towards a colonic leak (and obviously no leak was seen on CT scan and nor was fecal matter found!). If this is the clinical approach and attitude of today's doctors, I strongly feel we have a problem and that problem must be addressed. A landmark review article published in New England Journal of Medicine (NEJM)(Nov 2007) by David Brenner et al. from Columbia University Medical Center revealed that as many as one third of all CT scans in the United States are unnecessary. So the aim of this editorial is to spread awareness among doctors about the wise use of CT scans or at least to avoid over-enthusiasm about it. I would like to highlight the adverse effects of CT scans on four issues:

a. Radiation,

b. Contrast induced nephropathy (CIN),

c. Hypersensitivity to contrast mediums used in CT scan,

d. Cost factor.

\section{Editorial}

\section{Radiation}

The radiation dose of a typical single CT scan (eg. abdomen) is $10-15 \mathrm{mSv}$ (millisieverts), PET CT is around $25 \mathrm{mSv}$, and a typical chest X-ray is $0.01-0.02 \mathrm{mSv}$. Therefore, a typical CT scan imparts more than 500 times the radiation dose of a chest X-ray! Not only this, many patients ( $>30 \%$ ) undergo two or three CT scans which imparts $30-45 \mathrm{mSv}$ of radiation. A large cohort of Japanese atom bomb survivors who had radiation exposure ranging from $5-150 \mathrm{mSv}$ with a mean of $40 \mathrm{mSv}$, had a significant increase in cancer risk. The researchers from Columbia concluded that CT scans are responsible for $2 \%$ of all cancers in that country. There are other issues associated with CT scans like the newer CT scan machines are supposedly relatively 'radiation safe' than 
the older ones and there are even new studies which claim that radiation from CT scan is not significant. But findings of such studies will always pose a big question over their practical reliability. Also the 'old' CT scan machines having more radiation (and less definition) are still in use in many areas world over. Unfortunately many of the patients who had undergone scans from the older machines have to undergo repeat CT scans by new machines for want of more anatomical details. It is also to be noted that children are significantly more at risk for cancer by radiation.

\section{Contrast Induced Nephropathy (CIN)}

CIN is one of the leading causes of hospital- acquired acute kidney injury and CT scan is the leading cause of intravenous contrast media (IVCM) administration in patients. Though incidence of CIN is generally considered rare (about 2\% of people receiving dye can develop CIN), it is highly likely that the incidence of CIN is underreported. Also its incidence is significantly more in susceptible patients. Some important risk factors for CIN are:

- Renal insufficiency / other kidney disorders,

- Age $>60$ years,

- Diabetes Mellitus,

- $\quad$ Prior kidney surgery/single kidney,

- Hypertension requiring medical therapy,

- Circulatory insufficiency/shock secondary to trauma or other causes.

Though many of the CINs are reversible, some can lead to more serious problems involving both kidneys and systemic systems.

\section{Hypersensitivity to contrast mediums used in CT scan}

Many of the CT scan use contrast media which when used through intravascular route can cause allergic reaction ranging from mild to severe. Though the incidence is rare, many have died due to anaphylactic shock. Again there is high probability that such incidents are significantly underreported.

\section{Cost factor}

The cost of a CT scan is significant and by no means easily affordable by poor. Such investigations are definitely a problem/ burden for economically challenged people/countries. The problem of over use of CT scans has become an epidemic in our present society and it is going to worsen in coming years if appropriate steps are not taken now. This has become a complex issue with interplay of many factors leading to overuse of CT scans like:
Doctor factors: over-enthusiasm, ignorance, financial reasons, litigations etc. Many of these doctors practice 'defensive medicine' with the rationale "You are at fault if you miss anything." Unfortunately, most of the patients are not fully informed by doctors about the potential side effects of CT scans.

Patient and public factors: Now a day it has become common for patients demanding 'Full body CT scans' as these are often advertised openly. This is utter nonsense. This hype is mostly company driven. Also, there is a public myth that this scan will detect problems in their early stage and is an ultimate test for any bodily illnesses. No research supports the benefits or safety of full body CT scan. On the contrary, they are actively discouraged by reputed bodies like American College of Radiologists.

Absence/inaction of monitoring bodies: No clear guidelines / protocol are being followed in many countries / regions.

Company driven: It is no secret that both doctor and patient factors are influenced by companies/corporates.

The solution may not be easy but at least the following can be done:

a. Spread awareness among the doctors and the public.

b. Clear guidelines should be formed for doctors and companies.

c. Monitoring bodies should be active overlooking the activities of doctors and companies.

d. Laws should be made to defend doctors practicing evidence based medicine rather than defensive medicine.

The following simple protocol will help doctors in most of the situations (Figure 1).

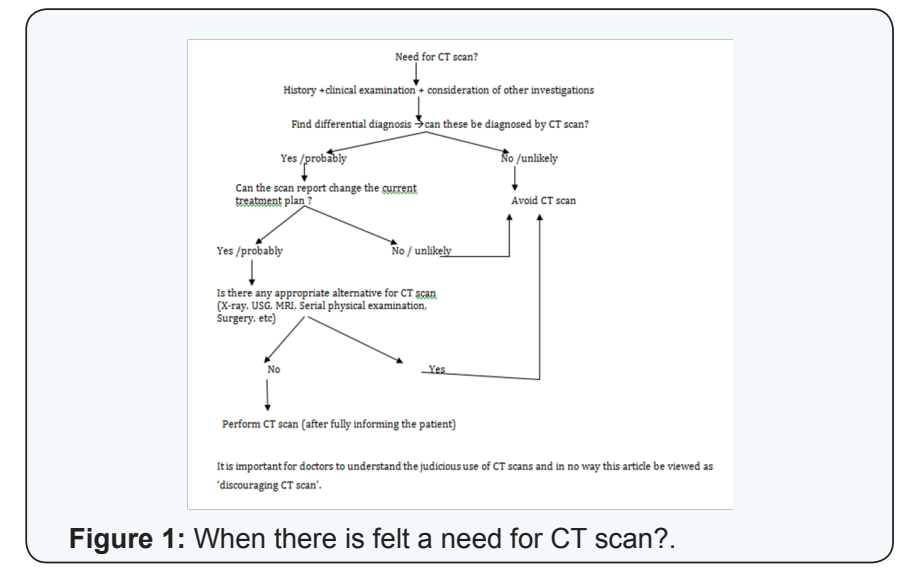

It is important for doctors to understand the judicious use of CT scans and in no way this article be viewed as 'discouraging CT scan'. 